\title{
Transcranial magnetic stimulation improves naming in Alzheimer disease patients at different stages of cognitive decline
}

\author{
M. Cotellia ${ }^{\mathrm{a}}$ R. Manenti ${ }^{\mathrm{b}}$, S. F. Cappa ${ }^{\mathrm{b}}$, O. Zanetti ${ }^{\mathrm{a}}$ and C. Miniussi $\mathrm{s}^{\mathrm{a}, \mathrm{c}}$ \\ ${ }^{\mathrm{a}}$ IRCCS S. Giovanni di Dio Fatebenefratelli, Brescia; ${ }^{\mathrm{b}}$ Department of Clinical Neurosciences, Vita Salute University, San Raffaele Scientific \\ Institute, Milan and National Neuroscience Institute, Italy; and ${ }^{\mathrm{c}}$ Department of Biomedical Sciences and Biotechnologies, University of \\ Brescia, Brescia and National Neuroscience Institute, Italy
}

\section{Keywords:}

dementia, language deficits, neurorehabilitation, repetitive transcranial magnetic stimulation

Received 3 January 2008 Accepted 7 May 2008
Objective: Word-finding difficulty (anomia) is commonly observed in Alzheimer's dementia (AD). The aim of this study was to assess the effect of repetitive transcranial magnetic stimulation (rTMS) applied to the dorso-lateral prefrontal cortex (dlPFC) on picture naming in 24 probable AD patients with different degrees of cognitive decline. Methods: High-frequency rTMS was applied to the left and right dlPFC during object and action naming in AD patients. A sham stimulation was used as a control condition. Results: Whilst, as previously reported, stimulation to both the left and the right dlPFC improved action, but not object naming in the mild AD group; an improved naming accuracy for both classes of stimuli was found in the moderate to severe group. Conclusions: Repetitive transcranial magnetic stimulation applied to the dlPFC improves naming performance also in the advanced stages of AD. Moreover, in the severe group the effect is not specific for action naming, as in the case of the mild AD group. These findings suggest that rTMS can affect the intrinsic ability of the brain to restore or compensate for damaged function and may represent an useful new tool for cognitive rehabilitation.

\section{Introduction}

In the cognitive neuroscience field, repetitive transcranial magnetic stimulation (rTMS) has been extensively aiming to map the flow of information across different brain regions and, in this respect, rTMS is a complementary technique for functional neuroimaging. Functional magnetic resonance, for example, can highlight the activation of a brain region whilst a given task is being performed, and rTMS can be used to check if that target area has a causal link with the required task.

However, rTMS effects are complex. In normal subjects, rTMS can modulate cortical excitability, improving or impairing cognitive performance, as it can transiently increase or decrease cortical activity depending on several stimulation parameters [1,2].

Repetitive transcranial magnetic stimulation can be applied either online (during task execution) or off-line (prior to task execution). Off-line rTMS is thought to induce long-lasting changes in cortical excitability, depending on a number of variables, such as frequency

Correspondence: Maria Cotelli, PhD, IRCCS S. Giovanni di Dio Fatebenefratelli via Piastroni 4, 25125 Brescia, Italy (tel.: + 39 0303501593; fax: + 390303501513 ; e-mail: mcotelli@ fatebenefratelli.it). stimulation, stimulus intensity, site of stimulation and number of applications. One of these parameters, the frequency of stimulation, is widely thought to be a critical determinant in the modification of cortical response. Both high- $(>5 \mathrm{~Hz})$ and low- $(\leq 1 \mathrm{~Hz})$ frequency rTMS have been employed, believing that the former has mainly an excitatory and the latter has mainly an inhibitory net effect [1]. Nevertheless, several studies have demonstrated that both types of stimulation (high and low frequency) may have similar, positive effects in selected pathological conditions, depending on the site of stimulation [3]. This finding is counterintuitive, as opposite changes in cortical excitability are expected to produce opposite effects, or in the best of cases, only one type of stimulation is expected to be effective. According to several authors [4], the crucial factor underlying these surprising results may be an imbalance in the functioning of the affected versus unaffected hemisphere. When applied over the affected hemisphere, high-frequency rTMS would be helpful in 're-establishing' its functions, whilst low-frequency rTMS would be effective only after stimulation of the unaffected hemisphere, as it reduces its hyperactivation which produces an increased transcallosal inhibition. 
In the presented study, we used rTMS during the execution of a task (i.e. online). Generally, experiments involving online rTMS provide two apparently contrasting results. In some cases (e.g. memory), rTMS can interfere with the task performance, inducing an increase in the number of errors [5]. In others (e.g. naming), even with comparable stimulation parameters, the performance can actually be 'facilitated' [6]. In general, these results underline the complexity of the effects of rTMS on behavioural performance, and they highlight the importance of an accurate selection of stimulation protocol and experimental task.

For example, using high-frequency rTMS in young normal subjects, Cappa et al. [6] reported a selective facilitation during verb naming when the subjects received stimulation to the left dorso-lateral prefrontal cortex (dlPFC). In the case of brain-damaged subjects, recent studies report improved ability to name pictures after administration of low-frequency rTMS to the anterior portion of the right homologue of Broca's area in patients with non-fluent aphasia [7-9]. In a recent study [10], we stimulated left or right dIPFCs during object and action naming in a group of Alzheimer's dementia (AD) patients. Action naming performance was improved in all subjects during high-frequency stimulation of both left and right dIPFC compared with sham stimulation. This was not the case for object naming, which was not significantly affected by rTMS.

The selective effect on action naming could be because of the specific contribution of dlPFC to this task, as predicted by theories that claim the existence of separate neural substrates for verb and noun processing and a crucial role of the dIPFC in action naming (see reviews in [11,12]). However, object naming is easier than action naming in early AD patients [13], and was largely unaffected in the mild AD patients examined in the previous study. The observed specificity may thus result from a ceiling effect for object naming.

The possibility that tasks involving verbs are simply more 'difficult' than task involving nouns seems to be refuted by the observation of a double dissociation between noun and verb processing, as well as by the lack of correlation between noun-verb performances discrepancy and the severity of language impairment of dementia [11]. Other hypotheses include the possible relationship of verb processing impairments with grammatical disorders, typically associated with frontal damage [14]; a link with executive dysfunction [15]; and the relation between verbs and action content [16]. Of particular note, Silveri et al. [15] found a stronger correlation between action naming deficit and severity of dementia in the frontal variant of fronto-temporal dementia (FvFTD) than in AD patients. The correlation analysis also suggested that the naming disorder was because of a dysexecutive deficit in FvFTD and linguistic disorder in AD.

The aim of the present study is to assess the effects of the high-frequency online rTMS procedure used in our previous study in AD patients in a more advanced stage of cognitive decline. In particular, we aimed to test the hypothesis of a selective effect of rTMS on action naming by applying the same procedure in severe AD patients, in which also object naming is typically affected. These data could clarify whether the absence of rTMS effects on object naming in mild AD underlined in our previous study could be explained simply by a ceiling effect. Alternatively, we could hypothesize that the selective effect on action naming highlighted in our previous study is because of the relevance of bilateral dIPFC for action but not object naming.

\section{Methods}

\section{Participants}

Twenty-four AD patients were recruited at the San Giovanni di Dio Fatebenefratelli Scientific Institute in Brescia, Italy. They were diagnosed as probable AD, based on criteria from the National Institute of Neurological and Communicative Disorders and Stroke Alzheimer's disease and Related Disorders Association (NINCDS-ADRDA) [17].

We divided the patients into two groups, mild (12) and moderate to severe (12), on the basis of the performance on MMSE [10]. Given that the overall group median was 17/30; we defined patients with a mild cognitive impairment as patients who had a MMSE $\geq 17 / 30$, whilst patients with a moderate to severe cognitive impairment were characterized by a MMSE $<17 / 30$. Demographic and clinical data of patients are reported in Table 1. The sample includes also the 15 patients reported in the previous study [18].

All patients were native Italian speakers and underwent a detailed clinical and neurological evaluation. All patients were on cholinesterase inhibitor (donepezil or rivastigmine) therapy and none of them was on memantine. For each patient, a structural brain MRI excluded major causes of cerebrovascular disease and

Table 1 Demographic and clinical data of AD groups

\begin{tabular}{lll}
\hline AD Group & Moderate to severe & \multicolumn{1}{l}{ Mild } \\
\hline$n$ & \multicolumn{1}{l}{12} & \multicolumn{1}{l}{12} \\
MMSE & $14.3 \pm 2.6$ & $19.7 \pm 1.6$ \\
Age (years) & $77.6 \pm 5.8$ & $75.0 \pm 6.2$ \\
Education (years) & $5.7 \pm 2.6$ & $6.8 \pm 3.1$ \\
\hline
\end{tabular}

AD, Alzheimer's dementia.

Data are expressed as mean $\pm \mathrm{SD}$. 
white matter lesions. MRI scans did not show focal atrophy, lacunae or severe subcortical vascular disease. All patients showed atrophy in medial temporal and cortical temporo-parietal regions, and four of 24 presented mild to moderate periventicular leukoaraiosis.

Patients with potentially confounding neurological and psychiatric disorders, clinically known hearing or vision impairment, a history of alcohol abuse, psychosis, or major depression were not included in the study. All the subjects had no implanted metal objects or history of seizure or heart problems. This exclusion criterion was based on the aim to keep stimulation as safe as possible [19]. The Local Human Ethics Committee approved the protocol.

A baseline evaluation of naming abilities was performed. The stimuli used in the action-object picture naming $(\mathrm{PN})$ task were taken from the Center for Research in Language International Picture Naming Project corpus (CRL-IPNP) [20], which contains 795 black and white 2D line drawings representing actions and objects. These items have been tested and normed in healthy and patient populations across seven different international sites and languages. Items were coded for a number of variables known to influence naming difficulty. Amongst others, these were: word frequency, age of acquisition and picture imageability scores.

For the baseline evaluation of naming abilities, we used a subset of 120 items from the original corpus. These were 60 actions and 60 objects. All the selected stimuli were high-imagery items. The nouns and verbs corresponding to the set of objects and actions were matched for word frequency and word length [6]. In this evaluation, AD patients showed a lower performance in action naming (accuracy: 53.44\%; SD = 17) than in object (accuracy: 72.66\%; SD = 16) naming.

\section{Experimental evaluation}

\section{Stimuli}

For rTMS task, we used a subset of 70 items from the original corpus, different from the 120 stimuli used for baseline testing. These were 35 actions and 35 objects. None of the actions included in the PN task were associated with the used objects. The nouns and verbs corresponding to the set of objects and actions were matched for target word frequency and length. Ten of the items were assigned to a practice block (five action and five objects), the remaining items were divided in three blocks designed for the three stimulation conditions. The frequency, length and grammatical category (noun or verb) of the target word were counterbalanced in the experimental blocks. Visual complexity and imageability of the pictures were also matched between the sets.

\section{Procedure}

Patients sat in front of a 17-inch monitor controlled by a personal computer running presentation software (http://www.neurobs.com). The patient task was to name as fast as possible, a picture presented on the monitor until the response was given. Verbal responses were recorded and digitized with the program Goldwave [Ver 5.12 (Goldwave Inc., St John's, NL, Canada), http://www.goldwave.com] at $44.1 \mathrm{kHz}$. The responses were then analyzed off-line for accuracy.

The experiment included three blocks corresponding to three stimulation sites: left, right dlPFC and sham stimulation. Each block contained an equal number of object and action pictures, presented in a random order, and the stimulation site (left, sham or right rTMS) was counterbalanced. The stimulation site for the sham condition was on $\mathrm{Cz}$ (10/20 EEG system), but the coil was positioned perpendicular to the scalp, thus ensuring that no magnetic stimulation reached the brain during the sham condition. For left and right dlPFC, the Talairach coordinates of cortical sites underlying coil were estimated for each subject by the SofTaxic Evolution Navigator system (E.M.S., Bologna, Italy). Using this system, we marked on the scalp a stimulation site above Brodmann area 8 (Talairach coordinates $X= \pm 35$, $Y=24, Z=48$, middle frontal gyrus - at about halfway between $\mathrm{F} 3 / 4$ and $\mathrm{F} 7 / 8$, respectively, 10/20 EEG system). This frameless stereotaxic neuronavigation system registered the relative positions of landmarks on the head and the stimulation site, which could be identified in the individual brain MRIs. The system consists of a graphic user interface and a 3D Fastrak digitizer (Polhemus Inc., Colchester, VT, USA) having four receivers and one stylus (Static accuracy: 0.03 inches root mean square (RMS) for the $X, Y$, or $Z$ position; 0.15 RMS for receiver orientation; resolution: 0.0002 inches per inch of transmitter and receiver separation; 0.025 orientation). Three of these receivers were placed solidly on the subject's head by means of a dedicated helmet to rule out the inaccuracy because of head movements. The fourth receiver was accurately positioned on the rTMS coil to measure its position $(X$, $Y$ and $Z$ Cartesian coordinates) and orientation (azimuth, elevation and roll). The stylus was instead used to register craniometric landmarks on the subject's head.

Furthermore, the SofTaxic Navigator system permits the computation of an estimated volume of MRIs of the head to guide the rTMS coil positioning in subjects for whom MRIs were unavailable. The estimated MRIs are automatically calculated by means of a warping procedure, by operating on a generic MRI volume (template) on the basis of a set of points digitized from the subject's scalp. These digitized points are used to 
compute a subsequent set of reference points which are analogous to a set of points pre-localized on the scalp of the template. Finally, the warping procedure is carried out using these two corresponding sets of reference points. With respect to individual MRIs, the mean ( $\pm \mathrm{SD}$ ) accuracy of the estimated MRIs is 4.06 ( \pm 1.54$) \mathrm{mm}$, comparable to the spatial resolution of rTMS at motor threshold intensity [21].

This method represents a good compromise amongst localization accuracy, high economic demands relating to neuronavigation devices and availability of the single-subject MRI. It should be underlined that we can compute the location of the coil with very high precision. This does not strictly imply that we know with the same precision the width of the brain areas directly or indirectly influenced by the magnetic field, independently of the presence of single subject MRI. Therefore, we can only assume that during our experiment, we were predominantly stimulating the estimated cortex site underlying the coil centre.

To stimulate the dIPFC, we used a 70-mm figure-ofeight coil and we placed the junction of the two coil wings above the marked point. rTMS was delivered for $500 \mathrm{~ms}$ from the onset of the visual stimulus using a frequency of $20 \mathrm{~Hz}$. We decided to stimulate for the first $500 \mathrm{~ms}$ with a frequency of $20 \mathrm{~Hz}$ because we were looking for a facilitation effect, as reported in the previous study [18,22]. The stimulation intensity used during the experiment was set at $90 \%$ of each subject's resting motor threshold. These parameters are in line with safety recommendations for rTMS [10] and none of the patients showed side effects of stimulation.

\section{Results}

Figure 1 shows the mean naming scores in each of the stimulation conditions, plotted separately for objects and actions in the two patients groups. Naming ability, measured as the performance in action and object naming in the sham condition, was not correlated to the raw MMSE score $(P>0.05)$ and to education $(P>0.05)$.

The results were analyzed with repeated measures ANOVA with Stimulus Category (Action and Object) and Site of Stimulation (sham, left and right) as withinsubject factors and Group (mild and severe cognitive impairment) as between-subject factors. The main effect of Group was significant $[F(1,22)=7.36 ; P=0.01)$, indicating that the two groups (mild and severe) were different if we consider overall naming abilities.

We then performed two separate repeated measures ANOVA for the two groups with Stimulus Category (Action and Object) and Site of Stimulation (sham, left and right) as within-subject factors.
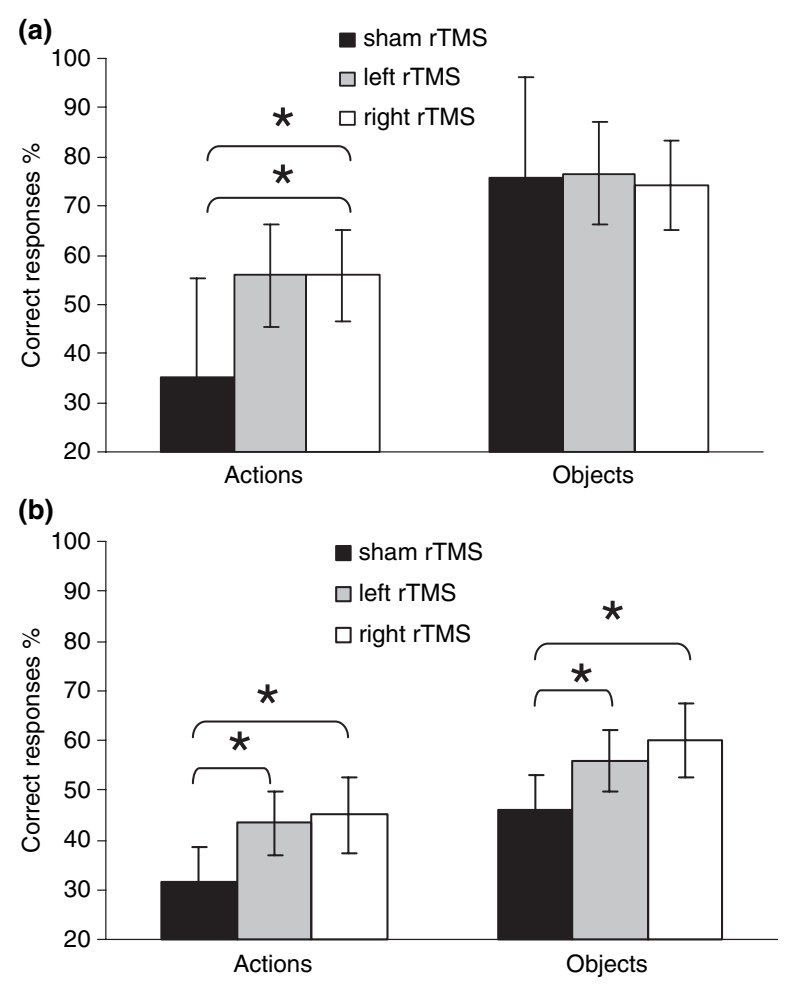

Figure 1 The graphs show the percentage of correct responses divided by stimulus category (action versus object), for different sites of repetitive transcranial magnetic stimulation (rTMS) in Alzheimer's dementia patients with MMSE $\geq 17$ (a) and in AD patients with MMSE $<17$ (b). ${ }^{*} P<0.05$.

For the mild group, the ANOVA indicated significant effects of both Stimulus Category $[F(1,11)=23.47$, $P=0.001]$ and Stimulus Category $\times$ Site of stimulation interaction $[F(2,22)=7.23, P=0.004]$. Post hoc analysis (with Bonferroni correction) revealed that for actions, naming performance was better during stimulation of both left $(P=0.002)$ and right $(P=0.002)$ dIPFC compared with sham stimulation. However, this was not true in the case of objects where performance did not differ significantly between conditions.

In the moderate severe patients group, the repeated measures ANOVA indicated significant effects of both Stimulus Category $[F(1,11)=7.822, P=0.03]$ and Site of Stimulation $[F(2,22)=7.48, P=0.004]$. Stimulus Category $\times$ Site of Stimulation interaction was not significant. Furthermore, post hoc analysis (with Bonferroni correction) revealed an improved accuracy both in action- and in object-naming during the stimulation of both left $(P=0.02)$ and right $(P=0.005)$ dlPFC, compared with sham stimulation.

Finally, a regression analysis was run to identify the predictor of naming improvement within each group. We performed a regression analysis on $\Delta$ Object and $\triangle$ Action, and MMSE score as regressor. The $\Delta$ value 


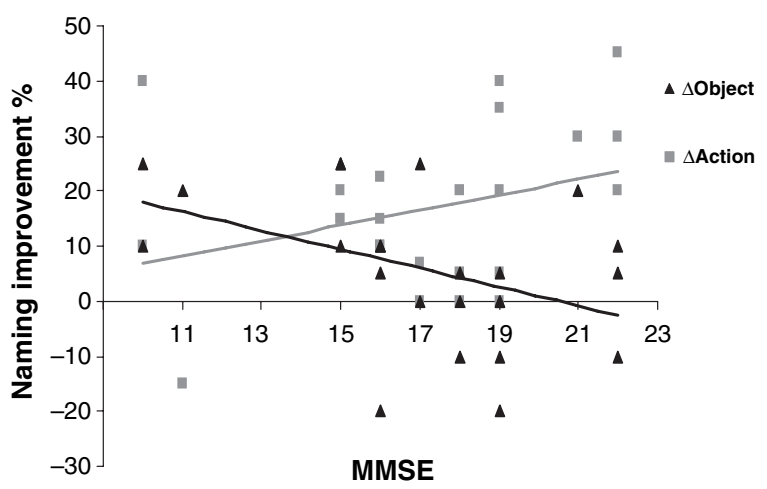

Figure 2 The graphs show regression analysis performed on $\Delta$ Object and $\triangle$ Action and regressor was MMSE score. Regression analysis was significant for $\triangle$ Object and MMSE score.

was calculated by subtracting sham score from object or action scores, respectively. As shown in Fig. 2, regression analysis was significant only for $\triangle$ Object and MMSE score $\left.\left[F(1,22)=4.988, P=0.03 ; R^{2}=0.18\right)\right]$. The regression analysis indicated that patients with a severe cognitive decline had an improved performance in object naming after rTMS compared with the performance of patients with a mild cognitive decline.

The distribution of the various error types pre- and post-stimulation was also analysed. The qualitative error analysis is reported in Table 2. Errors were classified into the following categories: semantically related errors (e.g. BICCHIERE, glass $\rightarrow$ BOTTIGLIA, bottle); semantically unrelated errors (e.g. BASTONE, walking stick $\rightarrow$ STRADA, street); phonologically related errors (e.g. TAVOLO, table $\rightarrow$ FAVOLO);

Table 2 Qualitative error analysis for action and object naming

\begin{tabular}{lllc}
\hline & $\begin{array}{l}\text { Sham } \\
\text { rTMS }\end{array}$ & $\begin{array}{l}\text { Left } \\
\text { rTMS }\end{array}$ & $\begin{array}{c}\text { Right } \\
\text { rTMS }\end{array}$ \\
\hline Actions (\%) & & & \\
Semantically related errors & 39 & 42 & 45 \\
Semantically unrelated errors & 26 & 29 & 30 \\
Phonologically related errors & - & - & - \\
Phonologically unrelated errors & - & - & - \\
Substitution with noun & 14 & 11 & 9 \\
Semantic error on stem & - & - & - \\
Anomia & 21 & 18 & 18 \\
Objects (\%) & & & \\
Semantically related errors & 58 & 58 & 58 \\
Semantically unrelated errors & 17 & 15 & 20 \\
Phonologically related errors & - & - & 2 \\
Phonologically unrelated errors & - & - & - \\
Substitution with noun & - & - & - \\
Anomia & 25 & 27 & 20 \\
\hline
\end{tabular}

rTMS, repetitive transcranial magnetic stimulation.

Results are expressed as mean of percentage of errors for each stimulation condition. There were no significant differences. phonologically unrelated errors (e.g. SEDIA, chair $\rightarrow$ FEBIE); substitution with noun (e.g. CASA, house $\rightarrow$ FIORE, flower); semantic errors on stem (e.g. CAMMINARE, to walk $\rightarrow$ PIEDERE foot + verb); and anomia. An ANova was performed with Stimulus Category (Action and Object), Site of Stimulation (sham, left and right) and type of error as factors. No significant results emerged from this analysis.

\section{Discussion}

The present findings indicate that rTMS to the dlPFC improves naming performance in AD patients not only in the early [7-9], but also in a more advanced stage of cognitive decline. Furthermore, they provide another direct evidence for a causal role of dlPFC in naming, in line with other recent studies on aphasic patients [6].

The same procedure, which in young control subjects shortened naming latency, resulted in an increased number of correct responses in AD patients. This suggests that the failure to observe an effect on performance accuracy in normal controls was because of a ceiling effect [6]. rTMS to the dIPFC had no effect on object naming either in normal subjects [10] or in mild $\mathrm{AD}$ patients [6]. However, it is important to highlight that naming latencies were shorter for objects than for actions in normal subjects [10], and that naming performance was higher with objects than with actions in mild AD patients [23,24].

In the present study, both object and action naming could be facilitated by dIPFC stimulation in moderate to severe AD patients. This result supports the interpretation that the lack of effects on object naming in our previous study was actually because of a ceiling effect, and that when object naming is impaired (i.e. moderate to severe AD patients), rTMS to the dIPFC results in an improved performance also for this class of stimuli.

Whilst the rTMS effect in normal controls was limited to the left-sided stimulation, the facilitation was bilateral both in mild and severe AD patients. The presence of a bilateral facilitation effect in AD patients could be attributed to the presence of a compensatory mechanism based on the recruitment of right hemispheric resources to support the residual naming performance. It has been shown that early in the course of the dementia, AD patients' brains retain a significant degree of functional plasticity $[25,26]$. A shift from unilateral to bihemispheric engagement has been repeatedly observed in healthy ageing as well as in dementia patients, in the case of memory tasks, and it has been suggested that it plays a compensatory role [27]. In the case of language, a crucial role in supporting performance after left hemispheric damage has been traditionally assigned to the right hemisphere [28]. 
Naming a picture is a complex process involving multiple components that depend on the function of several brain regions $[29,30]$. Word-finding difficulties are one of the earliest clinical manifestations of language impairment in AD. Several studies have demonstrated that the naming deficit in $\mathrm{AD}$ reflects inefficient access to semantic knowledge, rather than a true loss of semantic representations [31]. The improvement in performance observed after dIPFC stimulation may reflect a facilitation of lexical retrieval processes, suggesting that the naming disorder is because of defective access rather than semantic loss [32]. Studies of conditions, such as semantic dementia, in which semantic representations are considered to be degraded, are required to test the hypothesis of a specific effect of rTMS on access deficits [28].

The neurophysiological mechanisms responsible for rTMS-induced facilitation remain essentially unknown. There is no clear knowledge about spatial resolution of rTMS and, therefore, it is difficult to interpret the observed functional effects in terms of exact anatomical effects. The use of rTMS involves the discharge of a transient electromagnetic field through the skull. By means of rapidly changing magnetic fields, electric currents are induced in the brain and these, in turn, produce transynaptic depolarization of pools of neurons located in the superficial cortical layers [33]. In addition to these effects, it has been shown that rTMS can influence the activity of brain centres distant from the stimulated site and this is actually the starting point of clinical application of rTMS.

The result of this experiment may be explained by an activation of adjacent cortical areas that can be nonspecifically recruited to compensate the functional decline. This may be in line with the idea that compensation mechanisms are at play in supporting the defective performances of AD patients [23,24,34]. These mechanisms are generally in the direction of a reduction of functional specialization to counteract structural loss as a form of cortical plasticity [35]. Therefore, compensatory changes may be associated with recruitment of additional adjacent regions that are further activated by rTMS. Nevertheless, as said before, in addition to local effects, rTMS can influence the activity of brain centres distant from the stimulated site, presumably via cortico-cortical connections.

The capability of rTMS to interact with the intrinsic ability of the brain to restore or compensate for damaged function is a promise for possible applications in the field of cognitive rehabilitation. Although we do not expect that the type of experimental procedure used in the present experiment (high-frequency online rTMS) will have a lasting effect on naming (i.e. beyond the end of the trial to improve subsequent performance), we believe that the transient response to online rTMS may be a promising method for subject selection (responders) as well as for the development of stimulation protocols to be used for off-line rTMS as a possible rehabilitation tool.

\section{Acknowledgements}

We wish to thank patients and caregivers for their patience. This research was supported by a Project grant from the 'Ministero della Sanità' and from Associazione Fatebenefratelli per la Ricerca (AFaR).

\section{Disclosure}

The authors have reported no conflicts of interest.

\section{References}

1. Maeda F, Keenan JP, Tormos JM, Topka H, PascualLeone A. Interindividual variability of the modulatory effects of repetitive transcranial magnetic stimulation on cortical excitability. Experimental Brain Research 2000; 133: $425-430$.

2. Brignani D, Manganotti P, Rossini PM, Miniussi C. Modulation of cortical oscillatory activity during transcranial magnetic stimulation. Human Brain Mapping 2008; 29: 603-612.

3. Fitzgerald PB, Brown TL, Marston NA, et al. Transcranial magnetic stimulation in the treatment of depression: a double-blind, placebo-controlled trial. Archives of General Psychiatry 2003; 60: 1002-1008.

4. Murase N, Duque J, Mazzocchio R, Cohen LG. Influence of interhemispheric interactions on motor function in chronic stroke. Annals of Neurology 2004; 55: 400 409.

5. Miniussi C, Cappa SF, Sandrini M, Rossini PM, Rossi S. The causal role of the prefrontal cortex in episodic memory as demonstrated with rTMS. Supplements to Clinical Neurophysiology 2003; 56: 312-320.

6. Cappa SF, Sandrini M, Rossini PM, Sosta K, Miniussi C. The role of the left frontal lobe in action naming: rTMS evidence. Neurology 2002; 59: 720-723.

7. Martin PI, Naeser MA, Theoret H, et al. Transcranial magnetic stimulation as a complementary treatment for aphasia. Seminars in Speech and Language 2004; 25: 181191.

8. Naeser MA, Martin PI, Nicholas M, et al. Improved naming after TMS treatments in a chronic, global aphasia patient - case report. Neurocase 2005; 11: 182-193.

9. Naeser MA, Martin PI, Nicholas M, et al. Improved picture naming in chronic aphasia after TMS to part of right Broca's area: an open-protocol study. Brain and Language 2005; 93: 95-105.

10. Cotelli M, Manenti R, Cappa SF, et al. Effect of transcranial magnetic stimulation on action naming in patients with Alzheimer disease. Archives of Neurology 2006; 63: 1602-1604.

11. Cappa SF, Perani D. The neural correlates of noun and verb processing. Journal of Neurolinguistics 2003; 16: 183189. 
12. Shapiro K, Caramazza A. Grammatical processing of nouns and verbs in left frontal cortex? Neuropsychologia 2003; 41: 1189-1198.

13. Cappa SF, Binetti G, Pezzini A, et al. Object and action naming in Alzheimer's disease and frontotemporal dementia [see comment]. Neurology 1998; 50: 351-355.

14. Berndt RS, Mitchum CC, Haendiges AN, Sandson J. Verb retrieval in aphasia. 1. Characterizing single word impairments. Brain and Language 1997; 56: 68-106.

15. Silveri MC, Salvigni BL, Cappa A, Della Vedova C, Puopolo M. Impairment of verb processing in frontal variant-frontotemporal dementia: a dysexecutive symptom. Dementia and Geriatric Cognitive Disorders 2003; 16: 296-300.

16. Cotelli M, Borroni B, Manenti R, et al. Action and object naming in frontotemporal dementia, progressive supranuclear palsy, corticobasal degeneration. Neuropsychology 2006; 20: 558-565.

17. Folstein MF, Folstein SE, Mchugh PR. "Mini-mental state". A practical method for grading the cognitive state of patients for the clinician. Journal of Psychiatric Research 1975; 12: 189-198.

18. Wassermann EM. Risk and safety of repetitive transcranial magnetic stimulation: report and suggested guidelines from the International Workshop on the Safety of Repetitive Transcranial Magnetic Stimulation, June 5-7, 1996. Electroencephalography Clinical Neurophysiology 1998; 108: 1-16.

19. Bates E, Andonova E, D'amico S, et al. Introducing the CRL International Picture-Naming Project (CRL-IPNP). Center for Research in Language Newsletter, Vol. 12. La Jolla: University of California San Diego, 2000.

20. De Mauro T, Mancini F, Vedovelli M, Voghera M. Lessico Di Frequenza Dell'italiano Parlato (Spoken Italian frequency dictionary). Milan: Fondazione IBM Italia, 1994.

21. Herwig U, Schonfeldt-Lecuona C, Wunderlich AP, et al. The navigation of transcranial magnetic stimulation. Psychiatry Research 2001; 108: 123-131.

22. Machii K, Cohen D, Ramos-Estebanez C, Pascual-Leone A. Safety of rTMS to non-motor cortical areas in healthy participants and patients. Clinical Neurophysiology 2006; 117: 455-471.

23. Backman L, Andersson JL, Nyberg L, et al Brain regions associated with episodic retrieval in normal aging and Alzheimer's disease. Neurology 1999; 52: 1861-1870.
24. Becker JT, Mintun MA, Aleva K, et al. Compensatory reallocation of brain resources supporting verbal episodic memory in Alzheimer's disease. Neurology 1996; 46: 692700 .

25. Cabeza R, Anderson ND, Houle S, Mangels JA, Nyberg L. Age-related differences in neural activity during item and temporal-order memory retrieval: a positron emission tomography study. Journal of Cognitive Neuroscience 2000; 12: 197-206.

26. Rossi S, Miniussi C, Pasqualetti P, et al. Age-related functional changes of prefrontal cortex in long-term memory: a repetitive transcranial magnetic stimulation study. Journal of Neuroscience 2004; 24: 7939-7944.

27. Vandenbulcke M, Peeters R, Van Hecke P, Vandenberghe R. Anterior temporal laterality in primary progressive aphasia shifts to the right. Annals of Neurology 2005; 58: 362-370.

28. Deleon J, Gottesman RF, Kleinman JT, et al. Neural regions essential for distinct cognitive processes underlying picture naming. Brain 2007; 130: 1408-1422.

29. Manenti R, Repetto C, Bentrovato S, et al. The effects of ageing and Alzheimer's disease on semantic and gender priming. Brain 2004; 127: 2299-2306.

30. Nebes RD, Martin DC, Horn LC. Sparing of semantic memory in Alzheimer's disease. Journal of Abnormal Psychology 1984; 93: 321-330.

31. Paus T, Jech R, Thompson CJ, et al. Transcranial magnetic stimulation during positron emission tomography: a new method for studying connectivity of the human cerebral cortex. Journal of Neuroscience 1997; 17: 31783184.

32. Jefferies E, Baker SS, Doran M, Ralph MA. Refractory effects in stroke aphasia: a consequence of poor semantic control. Neuropsychologia 2007; 45: 1065-1079.

33. Heller L, Van Hulsteyn DB. Brain stimulation using electromagnetic sources: theoretical aspects. Biophysical Journal 1992; 63: 129-138.

34. Woodard JL, Grafton ST, Votaw JR, et al. Compensatory recruitment of neural resources during overt rehearsal of word lists in Alzheimer's disease. Neuropsychology 1998; 12: 491-504.

35. Cabeza R, Anderson ND, Locantore JK, Mcintosh Ar. Aging gracefully: compensatory brain activity in highperforming older adults. Neuroimage 2002; 17: 1394 1402. 